\title{
PRINCIPALES PROCESOS QUE IMPACTAN LA CALIDAD DEL AGUA PARA EL RIEGO EN POZOS COSTEROS DEL CENTRO SUR CUBANO
}

\author{
Processes impacting irrigation water quality in several coastal wells of Center-Southern Cuba
}

Carmen BETANCOURT AGUILAR ${ }^{1 *}$, Tania TARTABULL ${ }^{2}$, Yeny LABAUT ${ }^{3}$ y Roberto FERRADAZ ${ }^{4}$

${ }^{1}$ Centro de Estudios para la Transformación Agraria Sostenible, Universidad de Cienfuegos, carreta de Rodas km4, Cienfuegos Cuba

${ }^{2}$ Politécnico Felix Varela, carretera de Arimao, km 12, Cumanayagua Cienfuegos, Cuba

${ }^{3}$ Departamento de Gestión Ambiental, Centro de Estudios Ambientales de Cienfuegos, Calle 17 esq. Ave. 46 $\mathrm{s} / \mathrm{n}$, Reina, Cienfuegos, Cuba

${ }^{4}$ Recursos Hidráulicos, avenida 5 de Septiembre, Punta Gorga, Cienfuegos, Cuba

*Autora de correspondencia: carmenbetancouert@gmail.com

(Recibido febrero 2018; aceptado septiembre 2018)

Palabras clave: salinidad potencial, índice de permeabilidad, relaciones iónicas, actividades antrópicas

\section{RESUMEN}

La calidad del agua constituye una variable a controlar en la agricultura de regadío. El objetivo de la investigación fue identificar los principales procesos que impactaron la calidad del agua para el riego en pozos ubicados en sureste del litoral de la bahía Cienfuegos. Los datos proceden de la empresa Recursos Hidráulicos e incluye las concentraciones de los componentes mayoritarios y la conductividad eléctrica (CE) del agua de siete pozos, durante la etapa 1983-2015. Para facilitar el tratamiento se aplicó un análisis de similitud y se obtuvieron tres grupos de pozos con diferentes características físicas y químicas. La identificación de los procesos influyentes en cada grupo se logró mediante las relaciones iónicas y el análisis de componentes principales. En la caracterización del agua para el riego se usaron los indicadores: salinidad potencial (SP), índice de permeabilidad (IP), relación de adsorción del sodio (RAS), porciento de sodio posible (PSP), carbonato de sodio residual (CSR), CE, dureza total (DT), relación de Kelly (RK) y relación de adsorción del magnesio (RAM). El agua de los tres grupos fue bicarbonatada cálcica, con mayor proporción de estos iones en el primer grupo. Los principales procesos identificados fueron: lavado de la roca, intercambio catiónico, aporte de cloruro y sodio y la salinización de las aguas. El agua cumplió los requisitos establecidos para el riego. Sin embargo, en los pozos del segundo y tercer grupo se observó un incremento en la DT y una disminución del IP vinculado a actividades antrópicas desarrolladas en las cuencas.

Key words: potential salinity, permeability index, ionic relations, anthropogenic activities

\begin{abstract}
Water quality is a variable to be controlled in irrigated agriculture. The objective of the present investigation was to identify the main processes that impact water quality for irrigation in wells located in the southeast of the coast of Cienfuegos Bay. A database
\end{abstract}


from Hydraulic Resources enterprise was used, which included the concentrations of the major components and the electrical conductivity of the water of seven wells during the 1983-2013 period. To facilitate the data processing, a similarity analysis was performed that found three groups of wells with different physical and chemical characteristics. The identification of influential processes in each group was achieved through ionic relations and principal component analysis. In the characterization of water for irrigation, the following indicators were used: potential salinity (SP), permeability index (IP), sodium adsorption ratio, possible sodium percentage, residual sodium carbonate, electrical conductivity, total hardness $(\mathrm{TH})$, Kelly relation and adsorption ratio of magnesium. The water of the three groups was calcium carbonate, with a higher proportion of these ions in the first group. The main processes identified were: rock washing, cation exchange, chloride and sodium contribution and salinization of the waters. The water met the requirements for irrigation; however, in the wells of the second and third group, there was an increase in the $\mathrm{TH}$ indicator, with a decrease in the PI related to anthropogenic activities developed in the basins.

\section{INTRODUCCIÓN}

Uno de los factores importantes para el desarrollo de una región es la disponibilidad de fuentes de agua que reúnan los requisitos para varios propósitos, tanto en cantidad como en calidad. Es necesario considerar estos aspectos para un adecuado manejo del recurso (Choramin et al. 2015). Los recursos hidráulicos en todo el mundo están sometidos a enormes presiones debido al incremento de la demanda de agua con mejor calidad; esta demanda está condicionada por factores políticos, sociales y ambientales (Moreno y Roldán 2013). El desarrollo económico asociado a un incremento acelerado de la población ha generado una mayor demanda de agua para diferentes fines (Vasanthavigar et al. 2012).

La calidad del agua para el riego es de gran importancia por razones de seguridad, debido a su potencial efecto sobre la salud humana y de los ecosistemas en general (Graczik et al. 2011). Cuando el agua que se usa en el riego no reúne los requisitos pueden estar presentes organismos patógenos en vegetales como el tomate y la lechuga. El concepto de calidad de agua para este fin se refiere a las características que puedan afectar al suelo y a los cultivos en su uso a largo plazo (Bosch et al. 2012).

Para evaluar su calidad se deben definir criterios como la salinidad y la sodicidad (Ayers 1987, Asamoah et al. 2015). La salinidad depende del tipo y cantidad de sales disueltas, por lo que su medida incluye la determinación de la concentración de sales solubles totales, los iones calcio $\left(\mathrm{Ca}^{2+}\right)$, magnesio $\left(\mathrm{Mg}^{2+}\right)$, sodio $\left(\mathrm{Na}^{+}\right)$, potasio $\left(\mathrm{K}^{+}\right)$, sulfato $\left(\mathrm{SO}_{4}{ }^{2-}\right)$, cloruro $\left(\mathrm{Cl}^{-}\right)$, carbonato $\left(\mathrm{CO}_{3}{ }^{2-}\right)$, bicarbonato $\left(\mathrm{HCO}_{3}{ }^{-}\right)$y la conductividad eléctrica (CE) (Ayers y Wescot 1976, Arzola et al. 2013).
La sodicidad se determina en relación con la concentración relativa del $\mathrm{Na}^{+}$respecto a otros cationes. Con las variables surgidas de la evaluación de los criterios señalados anteriormente se calculan los indicadores que permiten determinar la aptitud del agua. Entre estos se incluyen la relación de adsorción de $\mathrm{Na}^{+}$(RAS), el carbonato de sodio residual (CSR), el $\mathrm{pH}$, la CE, el grado de acidez, el porcentaje de sodio posible (PSS), la salinidad efectiva (SE), la salinidad potencial (SP) y el índice de permeabilidad (IP) (Ayers 1987, Palancar 2006, Cortés-Jiménez et al. 2009, Lingaswamy y Saxena 2015).

En las comunidades costeras el agua subterránea constituye la principal fuente de abastecimiento para múltiples usos. Su calidad depende de factores tales como las características del suelo, la topografía de la región, las actividades humanas, entre otros (Zghibi et al. 2013). El riego puede modificar la calidad del agua de corrientes superficiales vecinas (Betancourt et al. 2012a) y al agua subterránea, por percolación de las aguas de lavado de áreas fertilizadas y tratadas con pesticidas.

Cuando los acuíferos costeros son explotados por encima de sus capacidades se rompe el equilibrio entre el agua subterránea y el agua de mar y ocurre la intrusión marina (Lin et al. 2012). El cambio climático afecta las condiciones hidrogeológicas, genera cambios en la temperatura del agua y en el balance de sales presentes, además favorece la intrusión marina (Kovalevskii 2007). Tanto la sobreexplotación de los acuíferos como las actividades antrópicas desarrolladas en las cuencas de alimentación y el cambio climático potencian la variación de la calidad del agua de los acuíferos costeros.

Para un manejo integral del agua en la agricultura es necesario conocer los principales procesos 
influyentes sobre su calidad. Existe un grupo de pozos localizados en el litoral del sureste de la provincia de Cienfuegos al centro-sur de Cuba que tienen diferentes usos, sin embargo, no se ha identificado los procesos que están influyendo en la composición y calidad del agua para el riego. El objetivo de la presente investigación fue identificar los principales procesos que impactaron la calidad del agua para el riego en estos pozos.

\section{MATERIALES Y MÉTODOS}

En el estudio se usaron los resultados de parámetros físicos y químicos de siete pozos ubicados al sureste de la provincia de Cienfuegos, en el tramo comprendido entre la desembocadura del río Yaguanabo hasta el este de la bahía de Cienfuegos (Fig. 1).

Los datos físicos y químicos y la información relacionada con el uso y explotación de los pozos durante la etapa de comprendida entre los años 1985 al 2015 se obtuvo en la Delegación Provincial de Recursos Hidráulicos (DPRH). El muestreo fue periódico pero los datos almacenados son irregulares y como resultado el número de mediciones fue diferente en cada pozo.
Para conocer la geología presente en las cuencas que alimentan los pozos se consultaron el Mapa Geológico a escala 1:50000 e información de archivo de la DPRH. La caracterización del suelo se hizo según Hernández-Jiménez et al. (2015) y sus usos mediante un mapa de la Delegación Provincial de la Agricultura en Cienfuegos que se corroboró con la información obtenida de recorridos por la zona y con entrevistas a directivos y pobladores del lugar.

La CE se midió por el método conductimétrico. Se usó como un indicador de medida de la salinidad porque indica el total de sales disueltas en el agua y se usa para determinar el daño producido por la salinidad (Tartabul y Betancourt, 2016). El Na+ y el $\mathrm{K}^{+}$se determinaron por fotometría de llama, el $\mathrm{Ca}^{2+}$ y el $\mathrm{Mg}^{2+}$ por el método volumétrico con el ácido etilendiamín tetracético. $\mathrm{El} \mathrm{HCO}_{3}{ }^{-}$y el $\mathrm{Cl}^{-}$por método volumétrico usando como patrón disoluciones diluidas de ácido sulfúrico y nitrato de plata. El $\mathrm{SO}_{4}{ }^{2-}$ se determinó por espectrofotometría, según la APHA (1980).

Los pozos con concentraciones similares en los componentes mayoritarios y la CE se identificaron mediante un análisis de agrupamiento jerárquico según la distancia euclidiana entre las variables. Dichas variables fueron previamente estandarizadas mediante

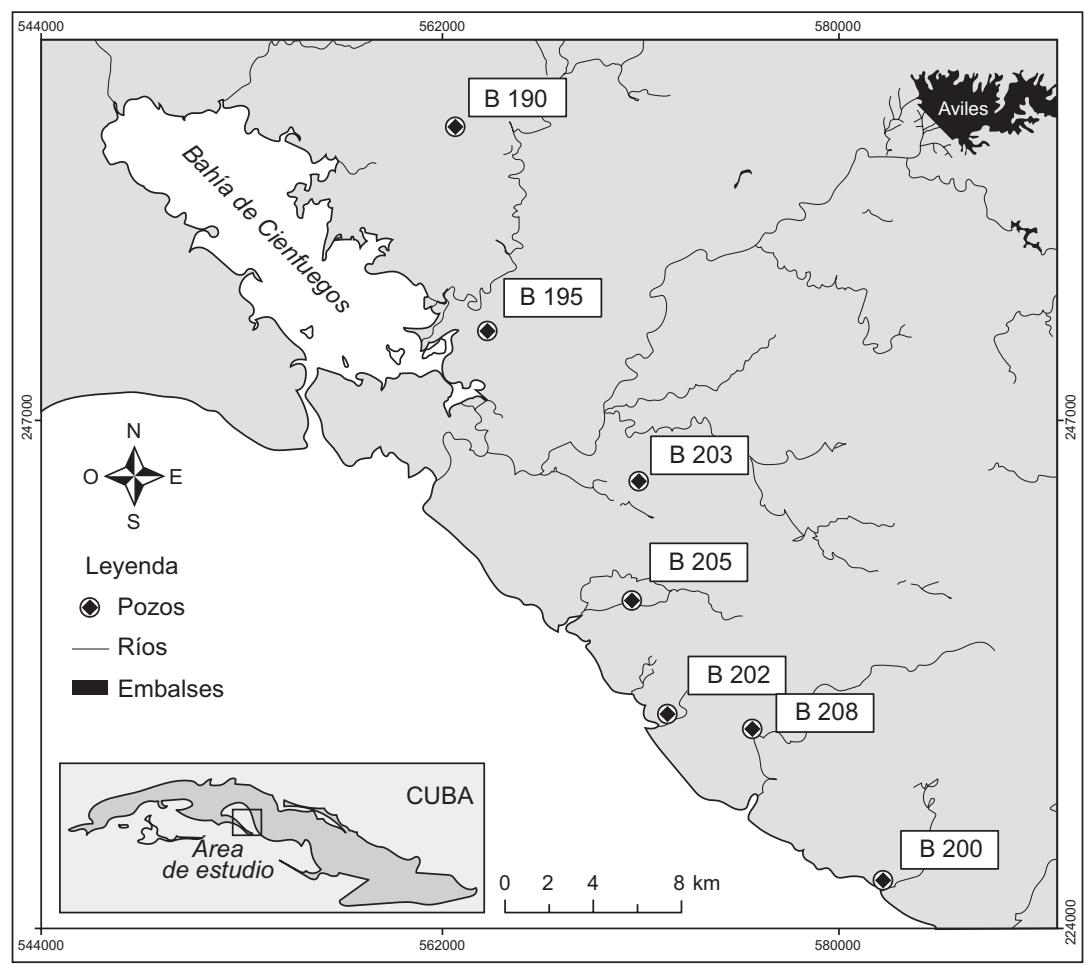

Fig. 1. Área de estudio. Se muestra la localización de cada pozo y las corrientes superficiales de la zona 
la expresión: $\log (\mathrm{x}+1)$. A cada grupo, determinado mediante el agrupamiento jerárquico, se le realizó un análisis descriptivo. Para encontrar las diferencias significativas de la CE y la proporción de los iones entre los tres grupos de pozos estudiados se aplicó un análisis de varianza (ANDEVA) por el método no paramétrico y según el criterio de Kruskal Wallis para $\mathrm{p} \leq 0.05$. Para realizar comparaciones pareadas entre las variables y poder establecer las diferencias se aplicó la prueba de Dunnett.

La identificación de los procesos influyentes sobre la composición de las aguas se realizó mediante el análisis de componentes principales (ACP). Se extrajeron tantos factores como autovalores mayores que la unidad tuvo cada grupo jerárquico identificado, lo cual permitió reducir las variables en estudio e identificar nuevas variables relacionadas con los procesos ocurrentes. Se usó la relación iónica entre el $\mathrm{Cl}^{-} \mathrm{y}$ el $\mathrm{Na}^{+}$(figuras donde la ordenada está representada por las concentraciones del ion $\mathrm{Cl}^{-}$y la abscisa por del ion $\mathrm{Na}^{+}$). Esta relación permite establecer cómo han sido los procesos geoquímicos en una cuenca determinada (Fernández y Miretzky 2004, Pehlivan y Yilmaz 2005, Rajmohan y Elango 2007, Betancourt et al. 2012b).

La aptitud del agua para el riego se determinó mediante las variables de mayor uso internacional y según los criterios de los autores que definieron dichas variables (Cuadro I). En el procesamiento estadístico así como en la construcción de las figuras para representar las relaciones iónicas se usaron las medias anuales de las variables correspondientes en cada grupo jerárquico. Los análisis estadísticos se realizaron con el paquete estadístico SPSS versión 15.
Para clasificar el agua según su contenido salino se usó el criterio de Arslan y Demir (2013) (Cuadro II).

CUADRO II. CLASIFICACIÓN DE LA SALINIDAD DEL
AGUA SEGÚN LOS VALORES DE LA CON-
DUCTIVIDAD ELÉCTRICA

\begin{tabular}{cc}
\hline Intervalo de valores & Criterio sobre la salinidad \\
\hline$<250 \mu \mathrm{S} / \mathrm{cm}$ & Baja \\
$250-750 \mu \mathrm{S} / \mathrm{cm}$ & Media \\
$750-2250 \mu \mathrm{S} / \mathrm{cm}$ & Alta \\
$\mathrm{CE}>2250 \mu \mathrm{S} / \mathrm{cm}$ & Muy alta \\
\hline
\end{tabular}

\section{RESULTADOS Y DISCUSIÓN}

\section{Caracterización de la zona de estudio}

La litología está constituida fundamentalmente por rocas sedimentarias (conglomerados, areniscas y calcáreas de granulometría diversa, caliza detrítica, de texturas brechosa y biógeno-arenosa) y rocas metamórficas como los esquistos, estratos delgados de mármol foliado y rocas eclogíticas. También están presentes rocas ígneas volcánicas como el basalto, andesitas, tobas y cuarzo.

Los pozos se localizan en el sureste de la Bahía de Cienfuegos en el centro-sur de Cuba a una altura sobre el nivel del mar que oscila entre los 4.49 y $36.87 \mathrm{~m}$. La zona es pobre en agua subterránea por lo cual se ubican próximos a corrientes superficiales vecinas. El agua extraída se usó en el riego agrícola, la cría de animales y el consumo humano.

CUADRO I. VARIABLES USADAS, ECUACIONES PARA SU DETERMINACIÓN Y AUTORES QUE DEFINEN LOS CRITERIOS DE LA CALIDAD DEL AGUA PARA EL RIEGO

\begin{tabular}{lll}
\hline Variable & Ecuación* & Autores \\
\hline SP & $\mathrm{SP}=\left[\mathrm{Cl}^{-}\right]+1 / 2\left[\mathrm{SO}_{4}^{2-}\right]$ & Balmaseda (2006) \\
IP & $(\mathrm{IP})=\left[\left(\mathrm{Na}^{2-}+\mathrm{H}_{2} \mathrm{CO}_{3}-\right) /\left(\mathrm{Ca}^{2+}+\mathrm{Mg}^{2+}+\mathrm{Na}^{+}\right)\right]^{*} 100$ & Balmaseda (2006) \\
RAS & $\left.\mathrm{RAS}=\left[\mathrm{Na}^{+}\right] /\left[\left(\mathrm{Ca}^{2+}+\mathrm{Mg}^{2+}\right) / 2\right]\right]^{1 / 2}$ & Richards (1954) \\
CSR & $\mathrm{CSR})=\left(\mathrm{CO}_{3}^{2-}+\mathrm{H}_{2} \mathrm{CO}_{3}^{-2}\right)-\left(\mathrm{Ca}^{2+}+\mathrm{Mg}^{2+}\right)$ & Richards (1954) \\
PSS & $\mathrm{PSS}=\frac{\mathrm{Na}^{+}+\mathrm{K}^{+}}{\mathrm{Ca}^{2+}+\mathrm{Mg}^{2+}+\mathrm{Na}^{+}+\mathrm{K}^{+}}$ & Wilcox (1955) \\
Dureza total & $\mathrm{DT}=(\mathrm{Ca}+\mathrm{Mg})^{*} 50(\mathrm{mg} / \mathrm{L})$ & Durfor y Becker (1964), \\
RAM & $\mathrm{RAM}=[\mathrm{Mg} /(\mathrm{Ca}+\mathrm{Mg})]^{*} 100$ & Ayers y Westcot, (1976) \\
RK & $\mathrm{RK}=\mathrm{Na} /(\mathrm{Ca}+\mathrm{Mg})$ & Kelly (1963) \\
\hline
\end{tabular}

*En las ecuaciones se usaron las concentraciones iónicas en meq/L. SP: salinidad potencial, IP: índice de permeabilidad: RAS: relación de adsorción del sodio, CSR: carbonato de sodio residual, PSS: porciento de sodio soluble: RAM: relación de adsorción del magnesio: RK: relación de Kelly. 
Los mayores volúmenes de extracción se realizaron en los pozos B203 y B205.

El suelo se usa en la cría de ganado, cultivos temporales y asentamientos poblacionales, de forma general, aunque en las áreas de alimentación del pozo B208 la actividad de riego estuvo deprimida. Tanto los asentamientos poblacionales como la cría de animales (principalmente ganado vacuno) constituyeron fuentes de contaminación para los pozos estudiados.

\section{Agrupamiento de los pozos según la similitud de sus componentes mayoritarios.}

El análisis de agrupamiento jerárquico de los pozos en estudio permitió la obtención de tres grupos: primer grupo compuesto por el pozo B208, el segundo grupo: B190, B195, B200 y B202, y el tercer grupo: B203 y B205. En el dendrograma se muestra en líneas discontinuas la estructura del grupo para la que hay similitud entre los pozos $(\mathrm{p}>0.05)$. Las líneas continuas representan las divisiones entre los pozos que fueron diferentes $(\mathrm{p} \leq 0.05)$ (Fig. 2).

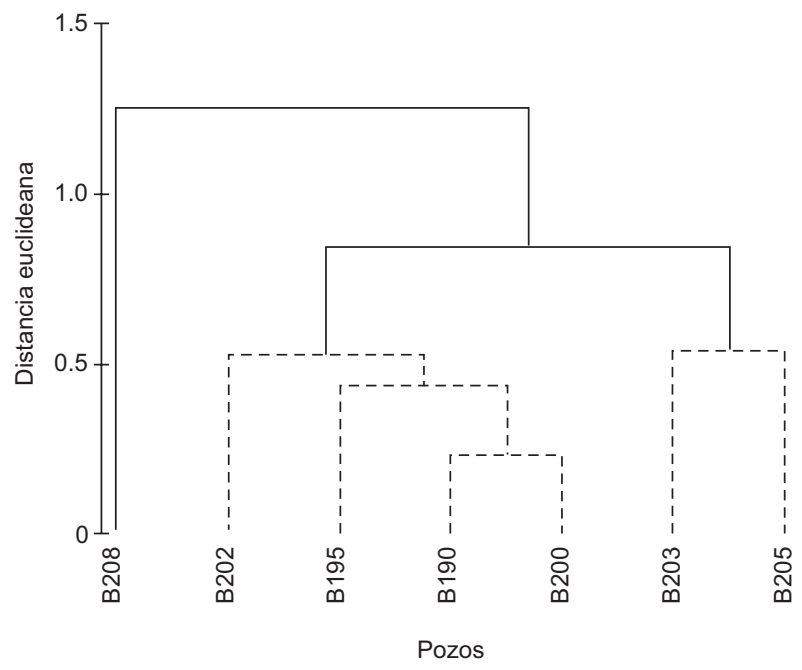

Fig. 2. Dendograma de distancias euclideanas entre pozos. Las líneas contínuas indican los grupos de pozos separados $(\mathrm{p}<0.05)$ mediante la prueba SIMPROF

\section{Composición física y química del agua de los pozos estudiados}

Los estadísticos descriptivos de los componentes mayoritarios del agua en cada uno de los grupos obtenidos mediante el dendograma revelaron que de forma general los valores más altos de los componentes mayoritarios y de la CE correspondieron a los pozos in- cluidos en el tercer grupo (B203 y B205)(Cuadro III). Se encontró diferencia significativa $(\mathrm{p} \leq 0.05)$ al comparar la $\mathrm{CE}$ y las proporciones de los iones de los tres grupos de pozos. Excepto las proporciones de los iones $\mathrm{SO}_{4}{ }^{2-} \mathrm{y} \mathrm{K}^{+}$, que fueron similares $(\mathrm{p}>0.5)$.

$\mathrm{La} C E$ fue más alta en el tercer grupo, seguido del segundo, mientras que el primero registró los valores más bajos $(\mathrm{p} \leq 0.05)$.

El agua se clasificó como bicarbonatada cálcica, sin embargo en la figura 3 se observa que ocurrió un incremento significativo en las proporciones de $\mathrm{Na}^{+}$ en el tercer y segundo grupo, con el valor más bajo para el primero $(p \leq 0.05)$. Una de las procedencias de estos iones son los aerosoles marinos (Jha et al. 2009). La cercanía de estos pozos a la zona costera pudo causar el incremento en las proporciones de $\mathrm{Na}^{+}$, ya sea por aerosoles o por intrusión, combinado con el uso y manejo de estas fuentes de agua que también hacen aportes. Al realizar esta misma comparación, las proporciones de $\mathrm{Ca}^{2+}$ y $\mathrm{HCO}_{3}{ }^{-}$fueron más altas en el primer grupo $(\mathrm{p} \leq 0.05)$. Cuando la composición salina del agua está determinada por la meteorización de las rocas, se caracteriza por un predominio de estos iones (Pehlivan y Yilmaz 2005, Jha et al. 2009).

\section{Identificación de los procesos que influyeron en la calidad del agua \\ Relaciones iónicas}

La relación establecida entre los iones $\mathrm{Cl}^{-}$y $\mathrm{Na}^{+}$ reveló que en el primer grupo de pozos estudiados el mayor número de observaciones se localiza encima de la línea 1:1, lo cual infiere concentraciones de $\mathrm{Cl}^{-}$superiores al $\mathrm{Na}^{+}$. En el resto ocurrió lo inverso (Fig. 4), cuando las concentraciones de $\mathrm{Na}^{+}$son superiores al $\mathrm{Cl}^{-}$se asocia a la influencia de procesos antrópicos sobre la calidad del agua (Giussani et al. 2008, Omo-Irabor et al. 2008, Jha et al. 2009).

Estos pozos se localizan en zonas de cultivos temporales donde aplican con sistematicidad el riego agrícola. También se localizaron áreas dedicadas a la cría de ganado y en el caso de los pozos del tercer grupo se extrajeron las mayores cantidades de agua, por lo que pudo ocurrir alguna penetración de aguas salinas procedentes del litoral. Resultados similares fueron encontrados en cuatro embalses cubanos afectados por este tipo de actividades antrópicas (Betancourt et al. 2012a).

\section{Análisis de componentes principales}

La aplicación del ACP a los tres grupos de pozos estudiados reveló que la medida de adecuación muestral de Kaiser-Meyer-Olkin (KMO) fue superior a 0.500 , la prueba de esfericidad de Bartlett fue 
CUADRO III. VALORES DE LOS ESTADÍSTICOS DESCRIPTIVOS DE LAS VARIABLES POR CADA GRUPO DE POZOS ESTUDIADOS. LACONDUCTIVIDAD ELÉCTRICA(CE) SE EXPRESA EN $\mu \mathrm{mhos} / \mathrm{cm}$ Y TODOS LOS IONES EN meq/L

\begin{tabular}{|c|c|c|c|c|c|}
\hline \multirow{10}{*}{$\begin{array}{l}\text { Primer grupo } \\
\text { Pozo } 208\end{array}$} & Variables evaluadas & Mínimo & Máximo & Media & Desviación estándar \\
\hline & $\mathrm{CE}$ & 401 & 786 & 487 & 66 \\
\hline & $\mathrm{pH}$ & 6.71 & 8.20 & 7.50 & 0.201 \\
\hline & $\mathrm{HCO}_{3}^{-}$ & 2.57 & 6.30 & 3.88 & 0.851 \\
\hline & $\mathrm{Cl}^{-}$ & 0.29 & 1.09 & 0.53 & 0.176 \\
\hline & $\mathrm{SO}_{4}{ }^{2-}$ & 0.05 & 2.11 & 0.34 & 0.346 \\
\hline & $\mathrm{Ca}^{2+}$ & 2.10 & 6.65 & 3.51 & 0.772 \\
\hline & $\mathrm{Mg}^{2+}$ & 0.38 & 2.42 & 1.01 & 0.484 \\
\hline & $\mathrm{Na}^{+}$ & 0.13 & 0.77 & 0.40 & 0.150 \\
\hline & $\mathrm{K}^{+}$ & 0.01 & 0.08 & 0.03 & 0.013 \\
\hline \multirow{9}{*}{$\begin{array}{l}\text { Segundo grupo } \\
\text { Pozos B190, } \\
\text { B195, B200 y B202 }\end{array}$} & $\mathrm{CE}$ & 435 & 1292 & 841 & 172 \\
\hline & $\mathrm{pH}$ & 6.58 & 8.03 & 7.36 & 0.211 \\
\hline & $\mathrm{HCO}_{3}^{-}$ & 3.55 & 9.69 & 6.09 & 1.020 \\
\hline & $\mathrm{Cl}^{-}$ & 0.38 & 2.93 & 1.38 & 0.560 \\
\hline & $\mathrm{SO}_{4}{ }^{2-}$ & 0.10 & 2.74 & 0.69 & 0.410 \\
\hline & $\mathrm{Ca}^{2+}$ & 3.00 & 8.80 & 5.68 & 1.360 \\
\hline & $\mathrm{Mg}^{2+}$ & 0.10 & 5.00 & 1.30 & 0.810 \\
\hline & $\mathrm{Na}^{+}$ & 0.31 & 4.00 & 1.59 & 0.778 \\
\hline & $\mathrm{K}^{+}$ & 0.01 & 0.95 & 0.07 & 0.092 \\
\hline \multirow{9}{*}{$\begin{array}{l}\text { Tercer grupo } \\
\text { Pozos B203 y B205 }\end{array}$} & $\mathrm{CE}$ & 620 & 1400 & 1022 & 163 \\
\hline & $\mathrm{pH}$ & 6.97 & 8.06 & 7.39 & 0.218 \\
\hline & $\mathrm{HCO}_{3}^{-}$ & 5.57 & 10.17 & 8.27 & 0.848 \\
\hline & $\mathrm{Cl}^{-}$ & 0.60 & 2.89 & 1.44 & 0.536 \\
\hline & $\mathrm{SO}_{4}^{2-}$ & 0.03 & 2.10 & 0.91 & 0.374 \\
\hline & $\mathrm{Ca}^{2+}$ & 2.60 & 7.40 & 5.25 & 0.789 \\
\hline & $\mathrm{Mg}^{2+}$ & 0.42 & 5.60 & 3.36 & 0.927 \\
\hline & $\mathrm{Na}^{+}$ & 0.18 & 4.45 & 2.34 & 0.940 \\
\hline & $\mathrm{K}^{+}$ & 0.00 & 0.55 & 0.07 & 0.092 \\
\hline
\end{tabular}

significativa $(\mathrm{p} \leq 0.05)$ y el determinante tuvo un valor aceptable para este análisis.

En el primer grupo de pozos (B208) se extrajeron tres componentes que explicaron el $73.9 \%$ de la varianza de los datos. El primer factor explicó el $34.5 \%$ (Cuadro IV) y se identificó como el aporte del lavado de la roca caliza a la composición química del agua. El segundo factor explicó el $24.5 \%$ y se relacionó con el lavado de rocas de silicatos y yeso. El tercer factor fue el que menos aportó con un valor de $14.9 \%$ y se relacionó con el aporte de fertilizantes que contienen $\mathrm{Cl}^{-} \mathrm{y} \mathrm{K}^{+}$. De estos resultados se infiere que la calidad del agua de este pozo está determinada fundamentalmente por procesos naturales (lavado de la roca o acción del intemperismo) representados por el $59 \%$ de la varianza total de los datos.

En el segundo grupo de pozos se extrajeron tres componentes que explicaron el $72.8 \%$ de la varianza de los datos, cada factor encontrado tuvo un peso similar $(24.7 \%, 21.5 \%$ y $21.5 \%)$ (Cuadro V).
El primer factor explicó el 24.7 \% y agrupó a la CE y los iones $\mathrm{HCO}_{3}{ }^{-}, \mathrm{SO}_{4}{ }^{2-}$ y $\mathrm{Mg}^{2+}$, que según la geología descrita, se encuentran presentes en las cuencas de alimentación de estos pozos. Este factor se identificó como el aporte de la meteorización de la roca.

El segundo factor explicó el $21.5 \%$ de la varianza de los datos y se relacionó con el aporte de cloruro de sodio $(\mathrm{NaCl})$ generado por la cría de ganado y las aguas de escorrentías procedentes de las áreas de riego. En la zona que alimenta estos pozos se localizan establos de la empresa pecuaria Sierrita, creada desde los años 80. En los últimos años la cría de ganado mayor asciende a 930 cabezas y se combina con la cría de ganado ovino y caprino. También se cultivan plátano, arroz y hortalizas, con alta frecuencia de riego. Varios autores (Giussani et al. 2008, Omo-Irabor et al. 2008, Jha et al. 2009) han informado resultados similares debido al aporte de residuales agropecuarios y escurrimiento de áreas de riego agrícola, a las aguas naturales. 

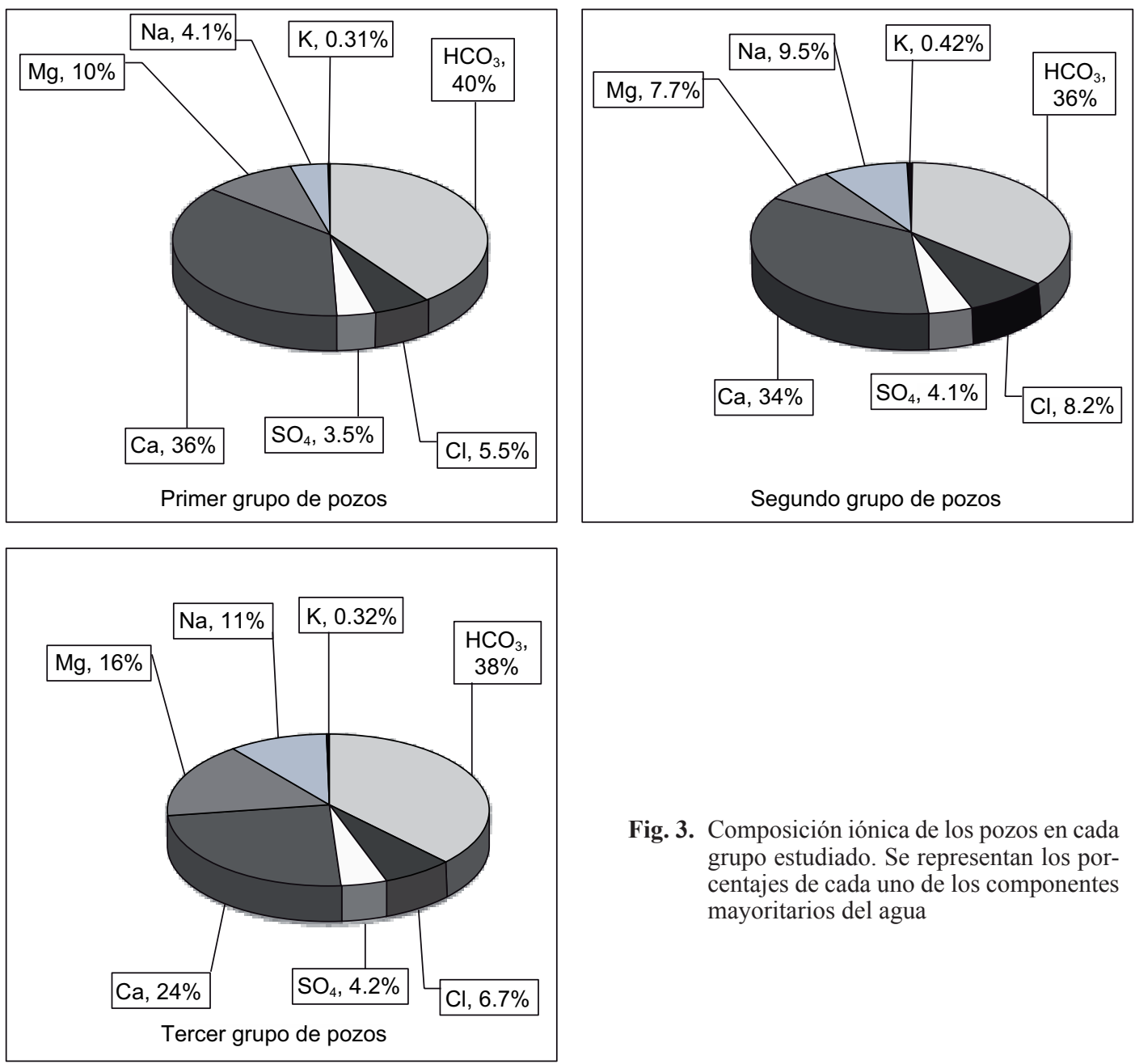

Fig. 3. Composición iónica de los pozos en cada grupo estudiado. Se representan los porcentajes de cada uno de los componentes mayoritarios del agua
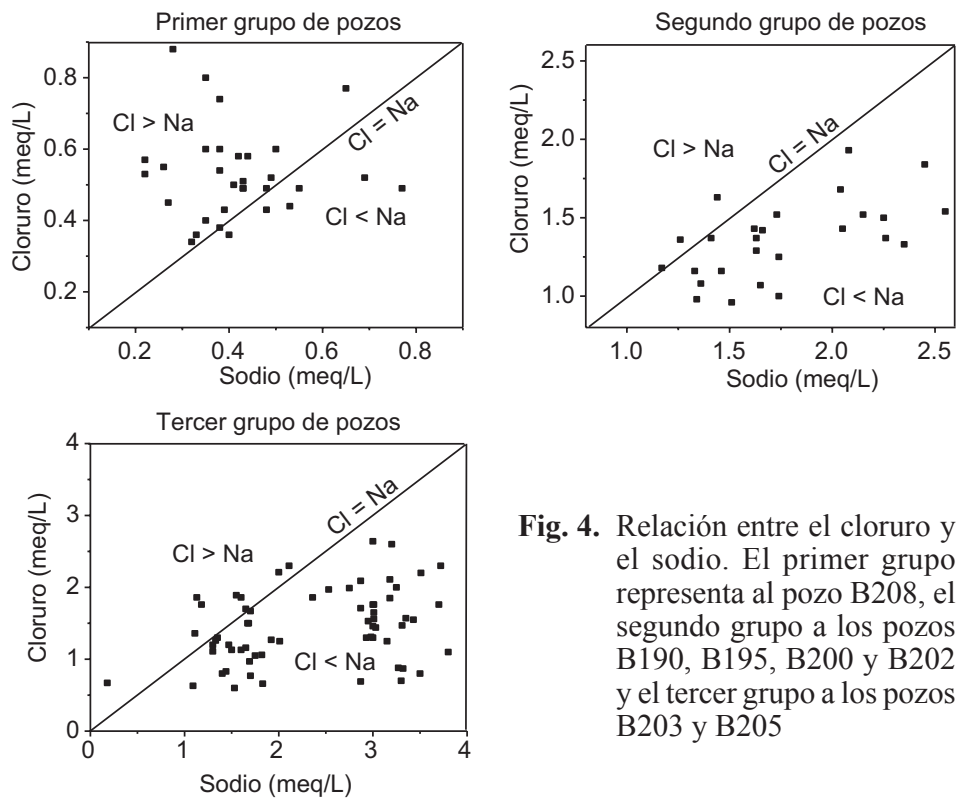

Fig. 4. Relación entre el cloruro y el sodio. El primer grupo representa al pozo B208, el segundo grupo a los pozos B190, B195, B200 у B202 y el tercer grupo a los pozos B203 y B205 
CUADRO IV. FACTORES DE CARGADE LAS VARIABLES MEDIDAS EN EL PRIMER GRUPO (POZO B208) PARA LOS TRES PRIMEROS EJES. LAS VARIABLES INCLUIDAS EN CADA COMPONENTE APARECEN EN NEGRITAS

\begin{tabular}{lrrr}
\hline \multirow{3}{*}{ Variables } & \multicolumn{3}{c}{ Componente } \\
\cline { 2 - 4 } & \multicolumn{1}{c}{1} & \multicolumn{1}{c}{3} \\
\hline $\mathrm{CE}$ & $\mathbf{0 . 8 0 9}$ & 0.368 & 0.089 \\
$\mathrm{HCO}_{3}{ }^{-}$ & $\mathbf{0 . 9 2 4}$ & -0.010 & -0.082 \\
$\mathrm{Cloruro}_{\mathrm{SO}_{4}{ }^{2-}}$ & -0.025 & 0.609 & $\mathbf{0 . 6 5 2}$ \\
$\mathrm{Ca}^{2+}$ & -0.219 & $\mathbf{0 . 6 6 6}$ & -0.442 \\
$\mathrm{Mg}^{2+}$ & $\mathbf{0 . 8 6 0}$ & 0.383 & 0.124 \\
$\mathrm{Na}^{+}$ & $\mathbf{- 0 . 5 4 1}$ & 0.531 & -0.006 \\
$\mathrm{~K}^{+}$ & 0.391 & $\mathbf{0 . 5 8 0}$ & -0.274 \\
& -0.116 & -0.493 & $\mathbf{0 . 6 8 8}$ \\
\hline
\end{tabular}

CUADRO V. FACTORES DE CARGA DE LAS VARIABLES MEDIDAS EN EL SEGUNDO GRUPO (POZO B190, B195, B200 Y B202) PARA LOS TRES PRIMEROS EJES. LAS VARIABLES INCLUIDAS EN CADA COMPONENTE APARECEN EN NEGRITAS

\begin{tabular}{crrr}
\hline & \multicolumn{3}{c}{ Componente } \\
\cline { 2 - 4 } Variables & \multicolumn{1}{c}{1} & \multicolumn{1}{c}{2} & \multicolumn{1}{c}{3} \\
\hline $\mathrm{CE}$ & $\mathbf{0 . 6 6 0}$ & 0.597 & 0.308 \\
$\mathrm{HCO}_{3}{ }^{-}$ & $\mathbf{0 . 8 1 8}$ & 0.215 & -0.010 \\
$\mathrm{Cl}^{-}$ & 0.123 & $\mathbf{0 . 8 7 4}$ & 0.197 \\
$\mathrm{SO}_{4}^{2-}$ & $\mathbf{0 . 6 1 3}$ & 0.081 & 0.344 \\
$\mathrm{Ca}^{2+}$ & 0.100 & 0.369 & $\mathbf{0 . 8 4 7}$ \\
$\mathrm{Mg}^{2+}$ & $\mathbf{0 . 7 7 8}$ & -0.071 & -0.466 \\
$\mathrm{Na}^{+}$ & 0.121 & $\mathbf{0 . 7 7 7}$ & -0.386 \\
$\mathrm{~K}^{+}$ & -0.003 & 0.245 & $-\mathbf{0 . 6 1 9}$ \\
\hline
\end{tabular}

El tercer factor explicó el $21.5 \%$ de la varianza de los datos y se vinculó al intercambio de bases. Esta nueva variable obtenida se relacionó de forma positiva con el $\mathrm{Ca}^{2+}$ y negativa con el $\mathrm{K}^{+}$. Parte del $\mathrm{K}^{+}$en solución puede retornar a la fase sólida y el $\mathrm{Ca}^{2+}$ pasa al agua (Chapman 1996). Este autor señala que el intercambio de bases es uno de los procesos que regula la composición química de las aguas (incorporación del $\mathrm{Ca}^{2+}$ y pérdida del $\mathrm{K}^{+}$).

En el tercer grupo de pozos estudiados se extrajeron dos componentes (Cuadro VI) que explicaron el $57.33 \%$ de la varianza de los datos, lo cual indica que existieron otros procesos que afectaron la calidad del agua que no pudieron identificarse mediante el ACP. El primer factor explicó el 39.7 \% y se identificó como el incremento de la salinidad del agua. Estos pozos se usan para el consumo humano (abastecimiento a las poblaciones de la Legua y Gavilán) y están más alejados del litoral que la mayoría de los incluidos en el segundo grupo, pero influyeron otros factores como el riego de cultivos, la cría de animales y la extracción de mayor volumen de agua. El pozo B203 está a $36.87 \mathrm{msnm}$, con una profundidad de $24.63 \mathrm{~m}$ y próximo al río Arimao en la zona estuarina. E1 B205 a 14.26 msnm y $6.12 \mathrm{~m}$ de profundidad se localiza en la zona estuarina del río Gavilanes.

CUADRO VI. FACTORES DE CARGA DE LAS VARIABLES MEDIDAS EN EL TERCER GRUPO (POZO B203 Y B205) PARA LOS DOS PRIMEROS EJES. LAS VARIABLES INCLUIDAS EN CADA COMPONENTEAPARECEN EN NEGRITAS

\begin{tabular}{ccr}
\hline & \multicolumn{2}{c}{ Componentes } \\
Variables & 1 & \multicolumn{1}{c}{2} \\
\hline Conductividad eléctrica & 0.921 & 0.140 \\
$\mathrm{HCO}_{3}^{-}$ & $\mathbf{0 . 7 8 3}$ & 0.018 \\
$\mathrm{Cl}^{-}$ & $\mathbf{0 . 5 4 2}$ & 0.188 \\
$\mathrm{SO}_{4}^{2-}$ & $\mathbf{0 . 7 2 1}$ & -0.046 \\
$\mathrm{Ca}^{2+}$ & 0.296 & $\mathbf{0 . 8 5 7}$ \\
$\mathrm{Mg}^{2+}$ & $\mathbf{0 . 5 8 2}$ & -0.481 \\
$\mathrm{Na}^{+}$ & $\mathbf{0 . 6 9 0}$ & -0.257 \\
$\mathrm{~K}^{+}$ & -0.017 & $\mathbf{0 . 5 6 5}$ \\
\hline
\end{tabular}

La extracción de altos volúmenes diarios pudo generar la entrada de agua con elevado contenido salino procedente de la zona estuarina. También es reconocido que el riego agrícola aumenta el contenido salino de las aguas receptoras (Rajmohan y Elango 2007, Miyamoto y Anand 2008, Betancourt et al. 2012 b) por el aumento en la concentración de sales en las aguas drenadas y la presencia de fertilizantes y pesticidas lixiviados, así como otros contaminantes en estado iónico.

En el cuadro III también se evidencia que a estos pozos le correspondieron los valores más elevados de la salinidad ( $\mathrm{p} \leq 0.05)$, expresada como CE. Al observar su composición química (Fig. 3) se evidencia una disminución en las proporciones de $\mathrm{Ca}^{2+}$ con un incremento en las de $\mathrm{Mg}^{2+}(\mathrm{p} \leq 0.05)$, que puede ser debido a la entrada de agua de la zona estuarina procedente de los ríos localizados en su cercanía. Wetzel (1975) refirió que en las zonas de los ríos cercanas a su desembocadura es característico encontrar una disminución en las proporciones de $\mathrm{Ca}^{2+}$ 
con un incremento en las de $\mathrm{Mg}^{2+}$. La escasa altitud en relación al nivel del mar que existe en la zona estuarina provoca la entrada de las aguas marinas hasta esa zona cuando ocurre marea alta. Es recomendable para pozos que se ubican cercanos a la zona litoral realizar las extracciones del agua cuando ocurre la marea baja.

El segundo factor explicó el $17.61 \%$ y se relacionó con disolución de rocas presentes en la cuenca. En la descripción litológica de estos pozos se pudo constatar que en el área circundante al pozo existe una zonación de las series volcánicas (rocas ígneas) que va desde las volcanitas toleíticas a la serie calcoalcalina (diferentes tipos de basalto; andesitas, dacitas o riolitas) con incremento de $\mathrm{Ca}^{2+} \mathrm{y} \mathrm{K}^{+}$.

\section{Determinación de la calidad del agua para el riego}

La evaluación de la calidad del agua para riego en el grupo uno (pozo B208) reveló que el agua reúne todos los requisitos para usarse en el riego agrícola. La RAS disminuyó significativamente en la etapa evaluada (Fig. 5), algo similar sucedió con los valores del PSS. Este pozo registró las concentraciones más bajas de los componentes mayoritarios y de la CE $(p \leq 0.05)$ (Cuadro III). El promedio de la CE clasificó el agua como de salinidad media, según el criterio usado y de buena calidad para el riego. Sin embargo, fue muy dura, lo cual puede interferir en el tiempo de vida de los sistemas de riego.

El análisis de los principales procesos influyentes en la calidad del agua informó que aproximadamente el $50 \%(49.4 \%)$ de la varianza de los datos se relacionaron con procesos naturales, y solo el $14.9 \%$ con actividades antrópicas (aporte de fertilizantes, aguas lixiviadas del riego, residuales pecuarios, entre otros), lo cual justifica el patrón observado en las variables evaluadas en este acápite. En este pozo la litología está representada por rocas ígneas, que son más resistentes a la meteorización y por tanto se justifica su menor contenido salino. Además en su cuenca de alimentación las áreas cultivables fueron menos extensas, por tanto la contribución de sales de las aguas de retorno que aportan las áreas de riego fue más escasa.

En el análisis del segundo grupo de pozos se verificó que sus aguas están aptas para el riego (Fig. 6). Sin embargo, el IP disminuyó de forma significativa durante la etapa de estudio, que aparentemente no concuerda con la disminución significativa del PSS. Esta falta de concordancia se justifica al observar el
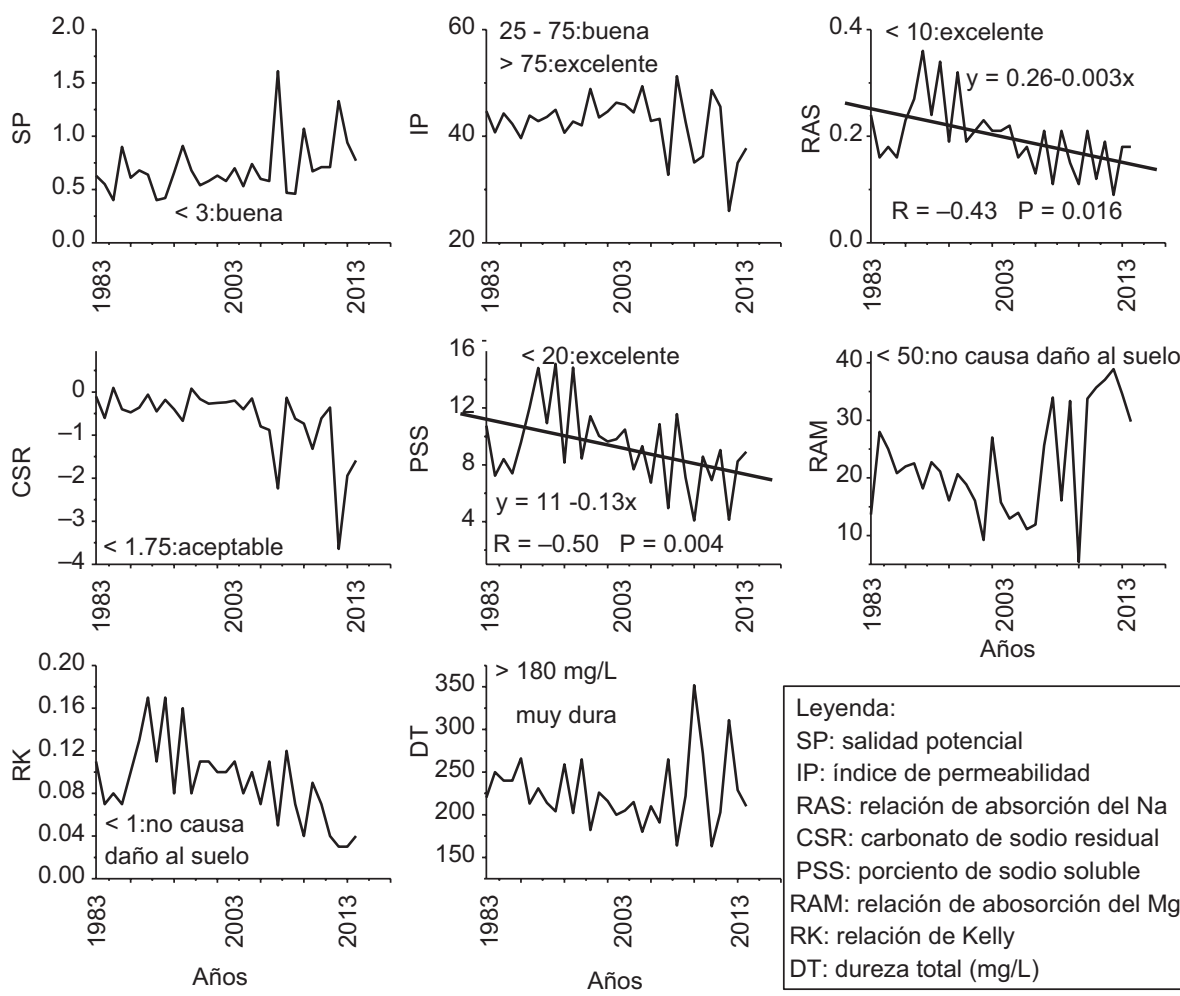

$$
\begin{aligned}
& \text { Leyenda: } \\
& \text { SP: salidad potencial } \\
& \text { IP: índice de permeabilidad } \\
& \text { RAS: relación de absorción del } \mathrm{Na} \\
& \text { CSR: carbonato de sodio residual } \\
& \text { PSS: porciento de sodio soluble } \\
& \text { RAM: relación de abosorción del Mg } \\
& \text { RK: relación de Kelly } \\
& \text { DT: dureza total (mg/L) }
\end{aligned}
$$

Fig. 5. Comportamiento de las variables usadas en la determinación de la calidad del agua para el riego en el primer grupo de pozos (B208) 

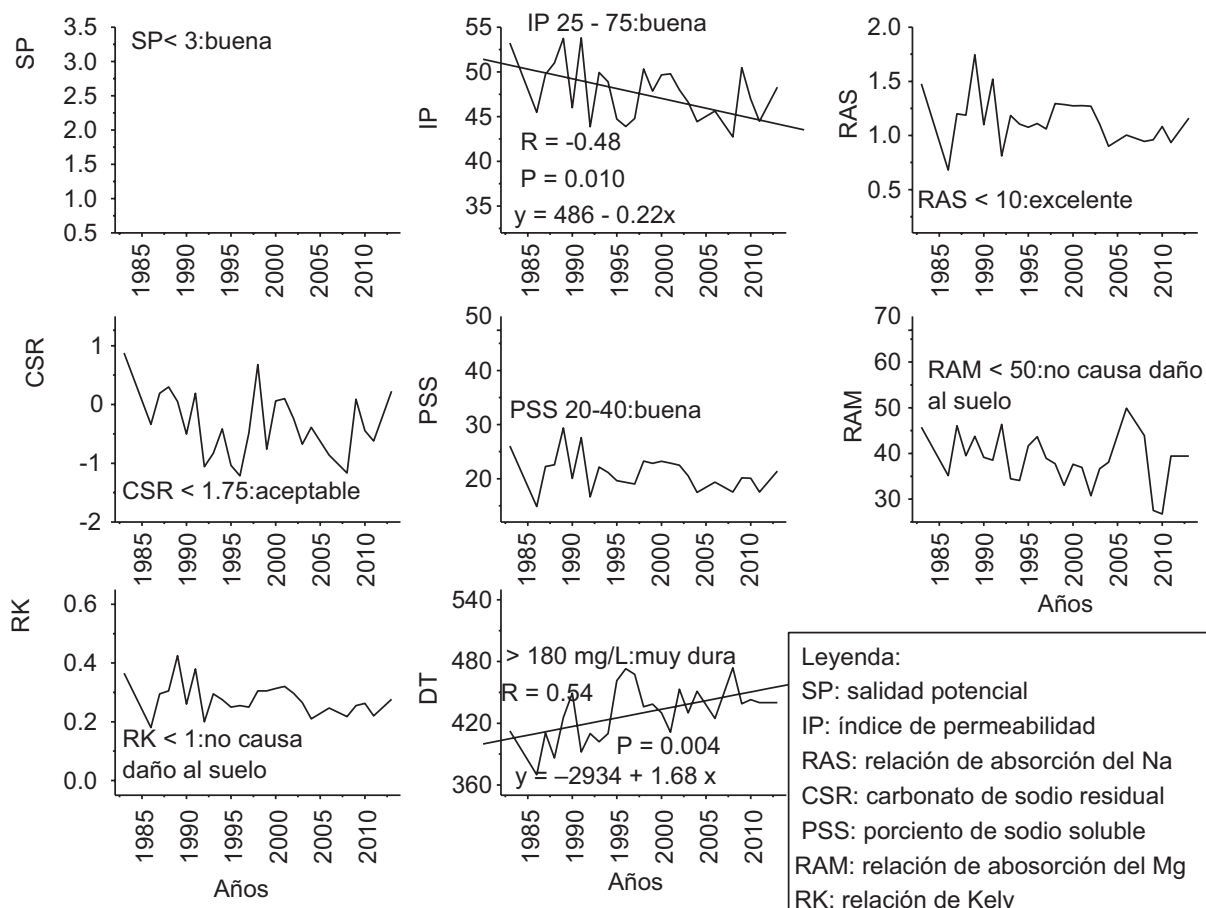

Leyenda:

SP: salidad potencial

IP: índice de permeabilidad

RAS: relación de absorción del $\mathrm{Na}$

CSR: carbonato de sodio residual

PSS: porciento de sodio soluble

RAM: relación de abosorción del Mg

RK: relación de Kely

DT: dureza total $(\mathrm{mg} / \mathrm{L})$

Fig. 7. Comportamiento de las variables usadas en la determinación de la calidad del agua para el riego en el tercer grupo de pozos (B203 y B205)

incremento de la DT, que generó un incremento en la proporción de $\mathrm{Ca}^{2+} \mathrm{y} \mathrm{Mg}^{2+}$, con la consecuente disminución del PSS. Los valores de la dureza clasificaron el agua como muy dura, lo cual va en detrimento del tiempo de vida útil de los sistemas de riego debido al depósito de las sales de Ca y $\mathrm{Mg}$. La CE según el criterio usado clasificó el agua como de alta salinidad y aceptable para el riego. El ACP aplicado a este grupo de pozos reveló que la segunda componente aportaba al $24.7 \%$ a la varianza de los datos y se relacionaba con el aporte de $\mathrm{Cl}^{-}$y $\mathrm{Na}^{+}$, lo cual justifica la disminución observada en el IP.

Los resultados obtenidos para el tercer grupo de pozos (B203 y B205), revelaron que todas las variables evaluadas señalan la buena calidad de estas aguas para el riego (Fig. 7), excepto la CE que clasifica al agua como de alta salinidad y aceptable para el riego (similar al segundo grupo de pozos). Las tendencias observadas en la DT y el IP, revelaron un deterioro en la calidad de sus aguas en el tiempo. La identificación de los procesos influyentes sobre la calidad del agua reveló que el mayor aporte a la varianza de los datos se verificó en la primera componente que se relacionó con la salinización del agua, lo cual concuerda con la tendencia a la disminución del IP.
Los resultados obtenidos al aplicar tres métodos diferentes para evaluar la calidad del agua y los procesos influyentes (la relación iónica, el ACP y la evaluación de la calidad del agua para el riego) constituyen una alerta temprana que permite trazar estrategias de manejo en relación al uso y explotación de estas fuentes de agua en el riego agrícola.

\section{CONCLUSIONES}

La aplicación del análisis de conglomerados permitió identificar tres grupos de pozos con características diferentes, lo cual facilitó la identificación de los principales procesos que impactaron la calidad del agua para el riego.

En los pozos B203 y B205 se extrajeron volúmenes de agua superiores al resto de los pozos, lo cual se relacionó con la salinización de sus aguas. El uso del suelo, las extracciones de agua y la litología de la cuenca de alimentación se relacionaron con la calidad del agua. Resultados similares se han encontrado en embalses cubanos en relación con la influencia del uso del suelo sobre la calidad del agua. 

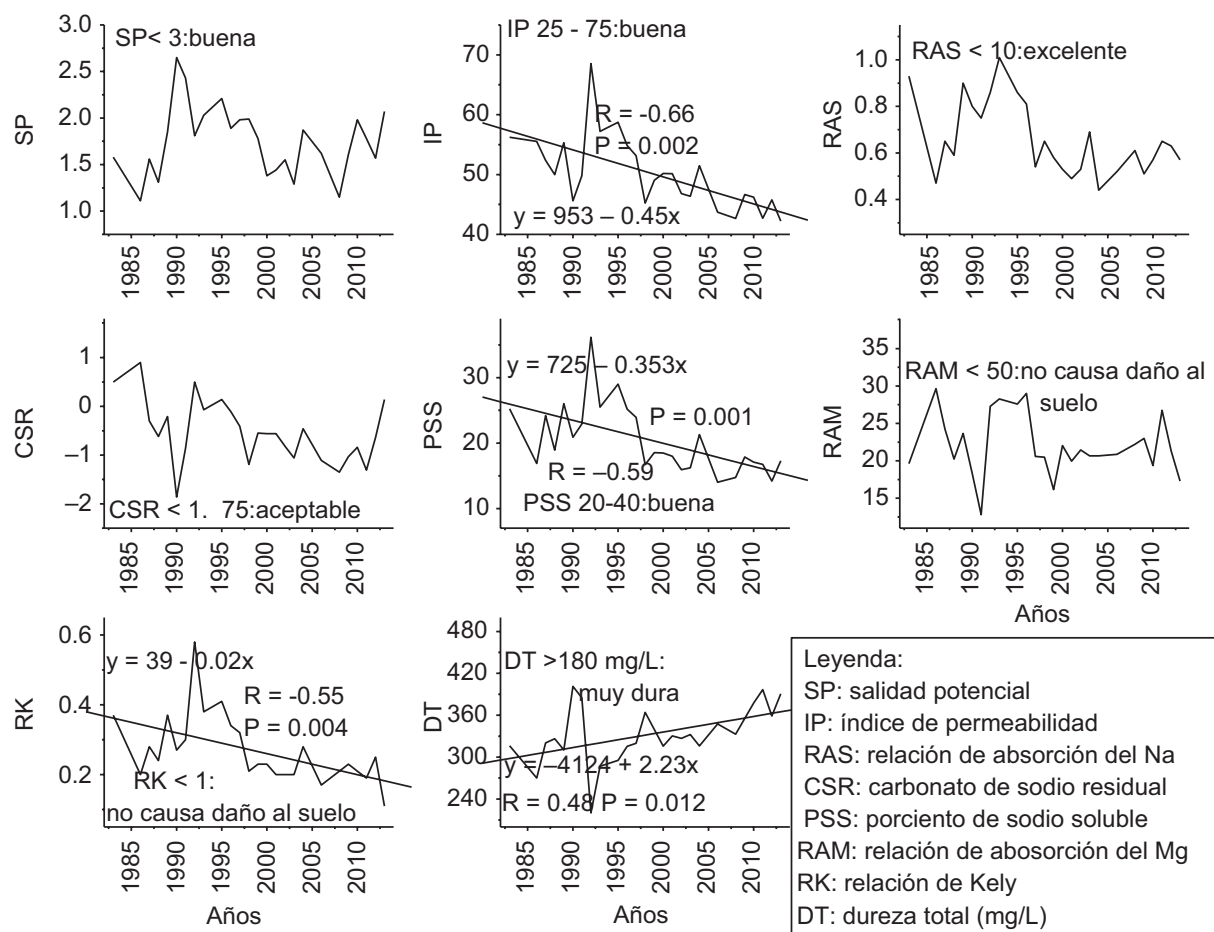

$$
\begin{aligned}
& \text { Leyenda: } \\
& \text { SP: salidad potencial } \\
& \text { IP: índice de permeabilidad } \\
& \text { RAS: relación de absorción del } \mathrm{Na} \\
& \text { CSR: carbonato de sodio residual } \\
& \text { PSS: porciento de sodio soluble } \\
& \text { RAM: relación de abosorción del Mg } \\
& \text { RK: relación de Kely } \\
& \text { DT: dureza total (mg/L) }
\end{aligned}
$$

Fig. 6. Comportamiento de las variables usadas en la determinación de la calidad del agua para el riego en el segundo grupo de pozos (B190, B195, B200 y B202)

Los principales procesos con influencia sobre la calidad del agua que fueron identificados son: lavado de la roca, intercambio catiónico, aporte de $\mathrm{NaCl}$ procedente de la cría de ganado y de las aguas de escorrentías procedentes de las áreas de riego, así como la salinización de las aguas.

Se observó una tendencia negativa en el IP, combinado con un incremento de la DT en el segundo y tercer grupo de pozos estudiados, lo que alerta sobre la posible afectación de la calidad del agua para el riego. Este deterioro fue más pronunciado en el tercer grupo donde el contenido de sales fue superior al resto.

\section{REFERENCIAS}

APHA(1980). Standard methods for the examination of water and wastewater. $15^{\text {th }}$ Edition. American Public Health Association, American Water Works Association, Water Pollution Control Federation, Washington, EUA, 1134 pp. Arslan H. y Demir Y. (2013). Impacts of seawater intrusion on soil salinity and alkalinity in Bafra Plain, Turkey. Environ. Monit. Assess. 185 (2), 1027-1040.

DOI: $10.1007 / \mathrm{s} 10661-012-2611-3$
Arzola N.C., Fundora O. y de Mello R. (2013). Manejo de suelos para una agricultura sostenible. Ed. Jaboticabal - Facultad de Ciencias Agrarias y Veterinaria/Universidad Estadual Paulista, Sao Paulo, Brasil, 511 pp.

Asamoah E., Nketia K.A., Sadick A., Asenso D., Kwabena E., Ayer J. y Owusu E. (2015). Water quality assessment of lake Bosomtwe for irrigation purpose, Ghana. Intl. J. Agri. Crop. Sci. 8 (3), 366-372.

Ayers R.S. y Wescot D.W. (1976). Calidad del agua para la agricultura. Organización de las Naciones Unidas para la Agricultura y la Alimentación. Roma, Italia, 85 pp.

Ayers R.S. (1987). La calidad del agua en la agricultura. Organización de las Naciones Unidas para la Agricultura y la Alimentación. Roma, Italia, 173 pp.

Balmaseda C., Ponce de León D., Martín N.J. y Vargas A.H. (2006). Compendio de Suelo. Universidad Agraria de la Habana, Cuba, 229 pp.

Betancourt C., Suárez R., Concepción E. y Herrera H. (2012a). Tendencia de los componentes mayoritarios del agua de cuatro embalses en el centro-sur cubano, durante un período de veinte años. Gestión Ambiental 23, 51-65.

Betancourt C., Suárez R. y Jorge F. (2012b). Influencia de los procesos naturales y antrópicos sobre la calidad del agua en cuatro embalses cubanos. Limnetica 31 (2), 193-204. 
Bosch M., Costa J.L., Cabria F.N. y Aparicio V.C. (2012). Relación entre la variabilidad espacial de la conductividad eléctrica y el contenido de $\mathrm{Na}+$ del suelo. Ciencia del Suelo 30 (2), 27-38.

Chapman D. (1996). Water quality assessments. A guide to use of biota, sediments and water in environmental monitoring. Second edition. Chapman y Hall, Londres, Inglaterra, $651 \mathrm{pp}$.

Choramin M., Safaei A., Khajavi S., Hamid H. y Abozari S. (2015). Analyzing and studding chemical water quality parameters and its changes on the base of Schuler, Wilcox and Piper diagrams (project: Bahamanshir River). WALIA Journal 31, 22-27.

Cortés-Jiménez J.M., Troyo-Diéguez E. y Murillo-Amador E. (2009). Índices de calidad del agua del acuífero del valle del Yaqui Sonora. Terra Latinoamericana 27 (2), 133-141.

Durfor C.N. y Becker E. (1964). Public water supplies of the 100 largest cities in the United States, 1962. American Water Works Association. Washington, EUA, 364 pp.

Fernández A. y Miretzky P. (2004). Ionic relations: a tool for studying hydrogeochemical processes in Pampean shallow lakes (Buenos Aires, Argentina). Quarter. Int. 114 (1), 113-121. DOI: 10.1016/s1040-6182(03)00046-6

Giussani B., Monticelli D., Gambillara R., Pozzi A. y Dossi C. (2008). Three-way principal component analysis of chemical data from Lake Como watershed. Microchem. J. 88, 160-166.

Graczik Z., Graczik T. y Naprauska A. (2011). A rol some food arthropods as vectors of human enteric infections. Center eur. J. Biol. 6 (2) 145-149.

DOI: $10.2478 / \mathrm{s} 11535-010-0117-\mathrm{y}$

Hernández-Jiménez A., Pérez-Jiménez J.M., BoschInfante D. y Castro-Speck N. (2015). Nueva Versión de Clasificación Genética de los Suelos de Cuba. La Habana, Cuba. Instituto de Suelos, 64 pp.

Jha P. K., Tiwari J., Singh U. K., Kumar M. y Subramanian V. (2009). Chemical weathering and associated $\mathrm{CO}_{2}$ consumption in the Godavari river basin, India. Chem. Geol. 264 (1-4), 364-374.

Kelly W.P. (1963). Use of saline irrigation water. Soil Sci. 95 (4), 355-391.

DOI: 10.1097/00010694-196306000-00003

Kovalevskii V.S. (2007). Effect of climate changes on groundwater. Water Res. 34 (2), 140-152.

Lin C.Y., Abdullah M.H., Praveena S.M., Yahaya A.H. y Musta B. (2012). Delineation of temporal variability and governing factors influencing the spatial variability of shallow groundwater chemistry in a tropical sedimentary island. J. Hydrol.432 (433), 26-42.

DOI: $10.1016 /$ j.jhydrol.2012.02.015
Lingaswamy M. y Saxena P.R. (2015). Water quality of Fox Sagar Lake, Hyderabad, Telangana State, India, Its Suitability for Irrigation Purpose. Int. J. Adv. Res. Sci. Technol. 4 (8), 490-494.

Miyamoto S. y Anand S. (2008). Hydrology, Salinity, and Salinity Control Possibilities of the Middle Pecos River: A Reconnaissance Report. Texas Water Resources Institute, Technical Report TR - 315. Texas, Estados Unidos. 31 pp.

Moreno F. y Roldán J. (2013). Assessment of irrigation water management in the Genil-Cabra (Cordoba, Spain) irrigation district using irrigation indicators. Agr. Water Manage. 120 (1), 98-106.

DOI: 10.1016/j.agwat.2012.06

Omo-Irabor O.O., Olobaniyi S., Oduyemi K. y Akunna J. (2008). Surface and groundwater water quality assessment using multivariate analytical methods: A case study of the western Niger Delta, Nigeria. Phys. Chem. Earth 33, 666-673.

Palancar T. (2006). Compresibilidad y resistencia al corte de suelos salinizados y sodificados por irrigación. Tesis de Doctorado. Universidad Nacional de la Plata, La Plata Argentina, $131 \mathrm{pp}$.

Pehlivan R. y Yilmaz O. (2005). Water quality and hydrogeochemical characteristics of the river Buyukmelen, Duzce, Turkey. Hydrol. Process. 19, 3947-3971.

DOI: 10.1002/hyp.5859

Rajmohan N. y Elango L. (2007). Hydrogeochemistry and its relation to groundwater level fluctuation in the Palar and Cheyyar river basins, southern India. Hydrol. Process. 20, 2415-2427. DOI: 10.1002/hyp.6052.

Tartabul T. y Betancourt C. (2016). La calidad del agua para el riego. Principales indicadores de medida y procesos que la impactan. Revista Agroecosistemas 4 (1), 47-61.

Vasanthavigar M., Srinivasamoorthy K., RajivGanthi R., Vijayaraghavan K. y Sarma V.S. (2012). Characterization and quality assessment of groundwater with a special emphasis on irrigation utility: Thirumanimuttar sub-basin, Tamil Nadu, India. Arab. J. Geosci. 5, 245-258.

Wetzel R. G. (1975). Limnology. W. B. Saunders Company. Philadelphia, EUA, 743 pp.

Wilcox L.V. (1955). Classification and use of the irrigation waters, Washington, D.C. Washington, EUA, 28 pp.

Zghibi A. Tarhouni J. y Zouhri L. (2013). Assessment of seawater intrusion and nitrate contamination on the groundwater quality in the Korba coastal plain of Cap-Bon (North-east of Tunisia). J. Afr. Earth sci. 87, 1-12. DOI: 10.1016/j.jafrearsci.2013.07.009 